\title{
IdeAs
}

Idées d'Amériques

$12 \mid 2018$

Le tourisme dans les Amériques

\section{L'écotourisme Vinte Quilos et le Projet intégré d'ethno-développement Waraná en Amazonie centrale brésilienne}

Vinte Quilos ecotourism and the ethno-development of the Waraná Project in the center of the Brazilian Amazon

O ecoturismo Vinte Quilos e o Projeto integrado de ethno-desenvolvimento

Waraná na Amazonia central do Brasil

\section{Bastien Beaufort}

\section{OpenEdition}

\section{Journals}

Édition électronique

URL : https://journals.openedition.org/ideas/3739

DOI : $10.4000 /$ ideas.3739

ISSN : $1950-5701$

Éditeur

Institut des Amériques

Référence électronique

Bastien Beaufort, «L'écotourisme Vinte Quilos et le Projet intégré d'ethno-développement Waraná en Amazonie centrale brésilienne », IdeAs [En ligne], 12 | 2018, mis en ligne le 27 février 2019, consulté le 19 octobre 2022. URL : http://journals.openedition.org/ideas/3739; DOl : https://doi.org/10.4000/ ideas.3739

Ce document a été généré automatiquement le 19 octobre 2022

\section{(c) (i) (2) $\Theta$}

Creative Commons - Attribution - Pas d'Utilisation Commerciale - Pas de Modification 4.0 International - CC BY-NC-ND 4.0

https://creativecommons.org/licenses/by-nc-nd/4.0/ 


\title{
L'écotourisme Vinte Quilos et le Projet intégré d'ethno- développement Waraná en Amazonie centrale brésilienne
}

\author{
Vinte Quilos ecotourism and the ethno-development of the Waraná Project in the \\ center of the Brazilian Amazon \\ o ecoturismo Vinte Quilos e o Projeto integrado de ethno-desenvolvimento \\ Waraná na Amazonia central do Brasil
}

Bastien Beaufort

1 Le centre d'écotourisme de Vinte Quilos est situé sur la bordure de la Terre Indigène Andirá-Marau. Celle-ci fut démarquée en 1984 puis homologuée en 1986 par le Décret 93.069 du 6 août 1986 au Brésil. Elle abrite 16000 habitants issus de nombreux clans appartenant au peuple Sateré Mawé. Ces derniers sont notamment connus pour avoir découvert le guaraná.

2 Le guaraná (Paullinia cupana Humboldt Bonpland Kunth var. sorbilis) est une liane sarmenteuse forestière de la famille des Sapindacées qui, d'après les chercheurs, fut domestiquée en arbuste cultivé il y a entre 2 et 4 millénaires (d'après Clement, Charles R. et al., $2010: 89$ \& Pereira N., $1954: 35)$ par les peuples Mawés. Le mot wara ná signifie en langue Sateré Mawé le principe de la connaissance. Devenu plante globale, le guaraná fait l'objet de filières multiples au Brésil et dans le monde ${ }^{1}$. L'une de ces filières, connue sous le nom du Projet Waraná, fut mise en place en 1994 par des producteurs et leaders Sateré Mawé afin de garantir un prix juste pour leur production de guaraná. Cette filière, qui dispose d'un site d'écotourisme, Vinte Quilos, s'inscrit dans le mouvement mondial du commerce équitable. Le commerce équitable est défini comme un mouvement international datant de l'après-guerre qui vise à améliorer les relations entre producteurs et consommateurs et à changer les règles du commerce mondial (voir le site de Commerce Équitable France, 2018). 
Depuis la fin des années 1990, le tourisme équitable et solidaire, plus souvent appelé écotourisme, est apparu comme un service qui applique les principes du commerce équitable aux échanges touristiques. L'écotourisme se présente ainsi comme un moyen de transformer les règles du tourisme conventionnel, notamment en termes d'équité entre les touristes et les hôtes et de respect de l'environnement (voir le site de l'Association pour le Tourisme Équitable et Solidaire - ATES, 2018). Dans le cas du Projet Waraná, le site d'écotourisme Vinte Quilos a pour objectif d'être une communauté vitrine du Projet intégré d'ethnodéveloppement des Indiens Sateré Mawé, qui s'articule notamment autour du commerce équitable du waraná. Au cours de cet article, nous présenterons l'écotourisme Vinte Quilos en Amazonie centrale en retraçant la genèse du Projet Waraná dont il fait partie, et dont il a vocation à être la vitrine.

Carte 1 : Localisation régionale de la Terre Indigène Andirá-Marau où se situe l'écotourisme de Vinte Quilos (Brésil)

Source : élaboration personnelle

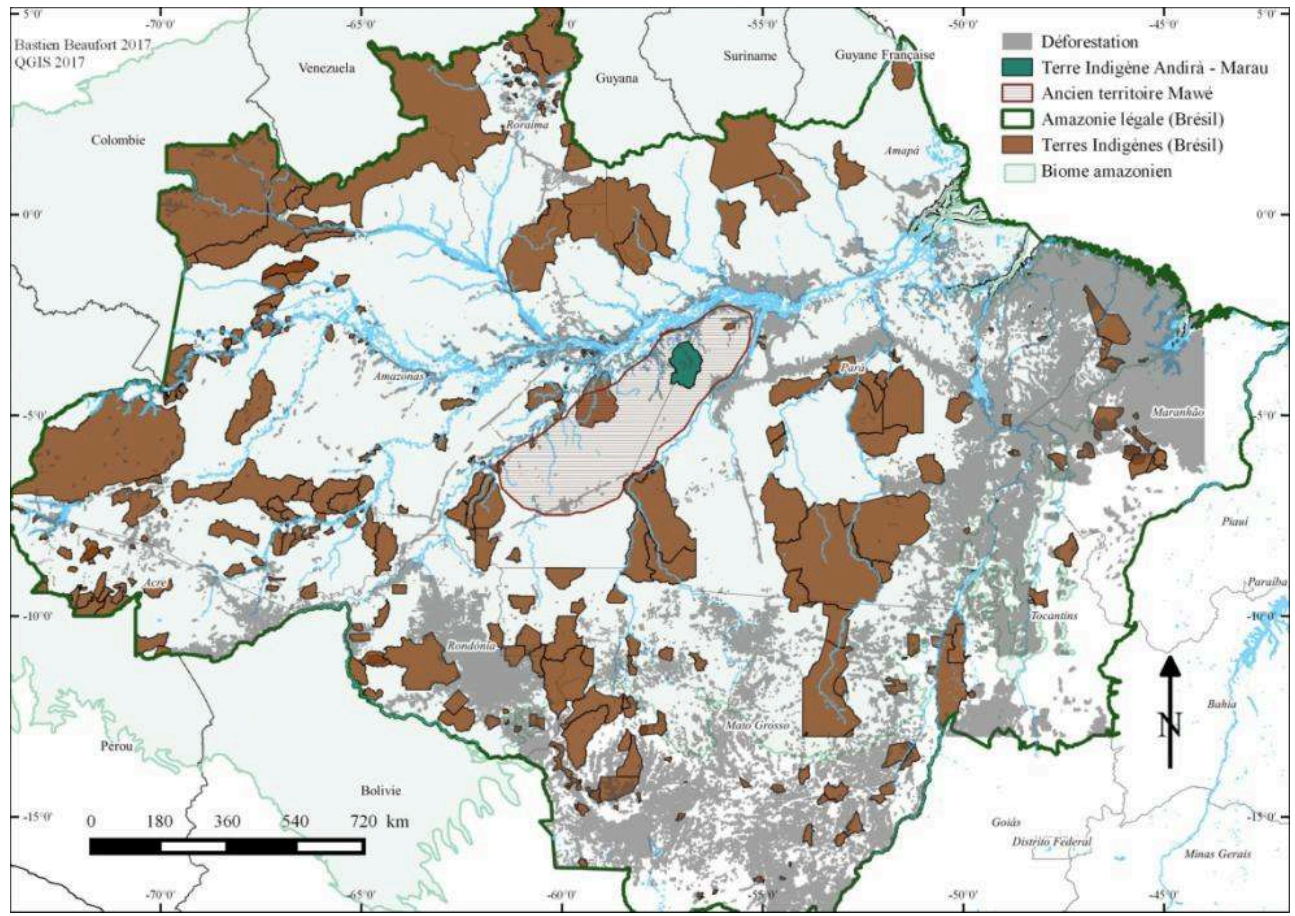

\section{Aux origines du Projet Waraná}

Le Projet Waraná est l'une des branches de la filière du guaraná où le commerce de la plante permet de soutenir l'autonomie d'« un peuple qui, grâce à toutes sortes de circonstances [...], a eu la chance de pouvoir survivre et de se définir aujourd'hui de manière autonome tant par rapport aux civilisations qui l'entourent que par rapport à son passé. » (Le Tourneau M., 2010 : 10). Comme tous les projets, le Projet Waraná est fondé sur un réseau, que Luc Boltanski et Eva Chiapello définissent comme des «multiplicités de rencontres et de connexions temporaires, mais réactivables, à des groupes divers, opérées à des distances sociales, professionnelles, géographiques et culturelles éventuellement très grandes.» (Boltanski L. \& Chiapello E., 1999: 157). Comme nous le verrons, cette 
définition s'applique à la lettre au Projet Waraná, dans sa constitution et sa genèse. Le Projet Waraná est aussi une filière économique en activité, dans la mesure où il repose sur le commerce du guaraná des Sateré Mawé, dénommé par eux waraná. Par ailleurs le Projet Waraná s'inscrit dans le réseau des communautés de la nourriture Terra Madre, idéalisé et mis en pratique par le gastronome Carlo Petrini.

Terra Madre est un réseau qui s'alimente au quotidien de ceux qui la composent, qui vit et s'enracine dans les territoires des communautés au niveau local. Ce réseau est en constante mutation et donc, à ce titre, très difficile à 'photographier' à un instant T. Ce qui en revanche peut être saisi, c'est ce qui l'unit, le ciment des valeurs qui demeurent incangées et hautement partagées (Petrini C., 2009 [2011] : 14-15)2 2.

Ces valeurs sont difficilement réductibles à une définition unifiée qui aurait le défaut de réduire le Projet Waraná à une seule de ses parties car, comme nous le verrons, ce dernier touche tant au commerce, qu'à la politique, à la mythologie et à l'épistémologie d'un peuple. Globalement, le Projet Waraná s'inscrit dans le courant de la socioéconomie associative. D'après Frédéric Debuyst, celle-ci est une modalité d'action dans le monde où

\begin{abstract}
L'action économique, s'encastre dans le socioculturel et que l'action solidaire ou associative couvre une pluralité de domaines, d'où notre dénomination de socioéconomie : l'associatif répond à la réunion de personnes sur une base plurifamiliale, d'une communauté, ethnie, région, quartier urbain, d'une similitude de conditions de vie, d'une convergence idéologique ou d'intérêts. Des acteurs multiples poursuivent une finalité commune, soit pour améliorer leur propre condition, soit en faveur d'autrui (Laville 2001). Le renforcement du lien social tend à l'emporter sur le souci du bénéfice matériel individuel. [...] L’idée que nous reprenons à divers auteurs serait qu'une SEA (socio-économie associative) devrait s'inscrire dans une économie plurielle, globale mais faite de pôles complémentaires, non exempts toutefois de contradictions et répondant à une logique autre que celle qui prévaut aujourd'hui dans les rapports entre économie et société (Evers 2000 ; Laville 2003). (Debuyst F., 2006 : 18)
\end{abstract}

Le Projet Waraná, s'il est symboliquement important à mettre en regard des filières conventionnelles de guaraná (Congrétel M., 2017), reste cependant modeste en termes de quantités de guaraná produites : moins de 20 tonnes annuelles, soit $0,004 \%$ à peine de la production brésilienne (Beaufort B., 2017). Pourtant, si le commerce équitable du waraná par les Indiens Sateré Mawé forme l'outil économique affiché de leur développement, ce commerce s'inscrit au service d'une politique d'ethnodéveloppement intégrée, qui trouve son origine dans les luttes amérindiennes foncières des années 1980 et leur organisation.

\title{
La création du Conseil Général de la Tribu Sateré Mawé (CGTSM)
}

7 Aujourd'hui, les Sateré Mawé représentent un peuple composé de 17000 habitants répartis entre la Terre Indigène Andirá-Marau (TI-AM, 14000 habitants) et les villes de Parintins, Barrheirinha, Maués et Manaus (3000 habitants). Au sein de la TI-AM les Sateré Mawé sont dispersés en près de 120 villages de quelques dizaines à 2000 habitants pour le plus gros d'entre eux. Ceux-ci sont disséminés sur les fleuves Andirá, Marau, Urupadi et Waicurapá. Le Projet Guaraná s'insère, chez les Sateré Mawé, au sein du principal organe politique du peuple: le Conseil Général de la Tribu Sateré Mawé (Conselho Geral da Tribo Sateré Mawé CGTSM). Ce dernier fut fondé, suite à la démarcation 
de la Terre Indigène Andirá-Marau et l'indemnisation d'Elf-Aquitaine-Braselfa ${ }^{3}$ le 15 septembre 1987 par le leader José Michiles dit Tuxaua Zuzu. «L'objectif de sa création était de construire pour le peuple Sateré Mawé un moyen de voir et de savoir choisir ce qui, dans le futur, serait bon pour la nation, et pour que nous ayons une protection vis-à-vis des exploiteurs. [...] A quoi servirait cette Organisation? A trouver des appuis et des soutiens pour les Sateré Mawé. » affirme-t-il dans une lettre qu'il laissa à sa mort (Wolf S., 2012). Cette organisation se base sur les articles 231 et 232 de la Consitution brésilienne qui fut promulguée en 1988, relatifs aux droits «Des Indiens» (dos Indios), qui leur accorde notamment l'usufruit des terres démarquées par l'Union. Le CGTSM est le conseiller du peuple Sateré Mawé, sa principale autorité et organisation qui institutionnalise dans un régime démocratique le pouvoir des tuxauas (twisa $)^{4}$.

Le CGTSM est Dieu, il est un instrument politique certes, mais il a une existence transcendantale. Il est difficile de sentir ou du moins de faire sentir cette réalité mais elle est profondément ancrée chez les Sateré Mawé. Elle met en évidence de manière quasi objective l'idée qu'ont les Sateré Mawé du Politique. Cette Idée est un grand sac où l'on trouve : les sehay wakuat / éthique du discours (bonnes paroles), le Guarana qui organise ce discours et qui est un conseiller, le Tuxaua qui est le conseiller, Jésus Christ et Dieu qui sont des conseillers (Isaïe 9.6). La Politique n'est alors pas la gestion de la population par la création de lois et le contrôle de leurs applications mais l'acte de conseiller le peuple. (Wolf S., 2012 : 103).

Photographie 1 : Le second drapeau du CGTSM avec le guaraná au centre (1994)

Source : Nusoken

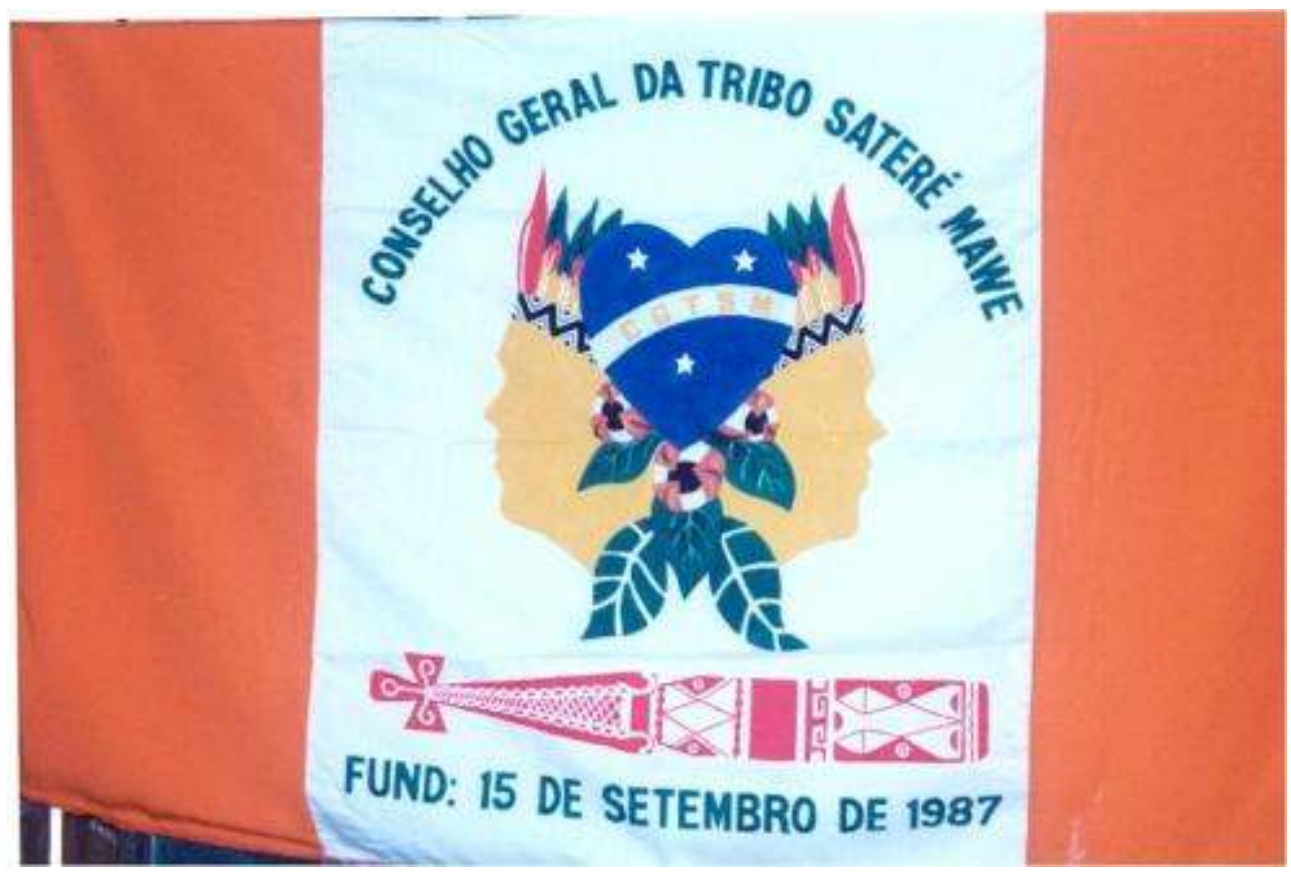

8 C'est à partir de ces événements politiques, et de la constitution de l'organisation politique des Indiens Sateré Mawé à travers le CGTSM, que le waraná fut identifié par les autorités traditionnelles autochtones (morekuat) et les nouveaux leaders comme un potentiel levier de développement alternatif. 


\section{La lente construction du Projet Waraná}

9 Twisa Donato ${ }^{5}$, un ancien meneur Sateré Mawé, raconte que l'idée de la valorisation du guaraná est née de la prohibition de la coupe du bois de rose, qui fut exploité de manière intensive sur les terres Sateré Mawé dans les années 1950 par des entreprises souvent transnationales notamment liées à l'industrie de la parfumerie.

Après l'interdiction du bois de rose, Dieu nous apporta un nouveau travail. Un jour, arriva le CGTSM. Cette association, CGTSM, parla au peuple. Aujourd'hui extraire du bois de rose est interdit. Pour cela, afin de gagner de l'argent, vous devez planter du manioc, de la patate douce, des bananes et autres. A l'époque de la fondation du CGTSM par le tuxaua Zuzu le guaraná n'avait pas de valeur. A cette époque j'en ai planté dans mon guaranazal, mais je laissais quelqu'un d'autre en prendre soin (abonar), car il n'avait pas de valeur. Il resta dans le désert (cerrado) et le guaraná sécha. Parce qu'à cette époque, personne ne voulait acheter du guaraná. Durant trois ans, il n'y eût aucun prix pour le guaraná (não houve preço de guaraná). Donc nous nous réunîmes à ce moment avec tuxaua Zuzu. Le CGTSM était alors déjà légalisé. Quand nous fîmes la réunion, nous discutîmes à propos du travail. Qu'estce qui serait le mieux à valoriser pour nous ? Le manioc, la patate douce, la canne à sucre. A ce moment nous commençâmes à penser à cela avec le tuxaua Servo. Alors nous avons dit: 'Le mieux serait de trouver un bon prix pour le guaraná.'. Alors nous fîmes deux réunions, mais nous n'y arrivâmes pas. Alors ensuite nous fîmes encore une fois une réunion et c'est à ce moment qu'arriva Obadias avec moi et dit : 'Avez-vous trouvé quelques personnes pour trouver un prix au guaraná, des personnes courageuses ?'. Quand il parla ainsi, le tuxaua Servo me tapota et me dit : 'Je crois que lui, il a de la volonté !' 'Alors nous allons le choisir lui pour trouver un prix pour le guaraná.' Grâce à cela, nous avons placé Obadias pour chercher un prix pour notre guaraná. Alors il s'en fut le chercher. En lui, nous trouvâmes un homme courageux (yt gen $\hat{e} i$ hat). C'est pour cela qu'aujourd'hui, nous avons un prix du guaraná au milieu de nous. (Tuxaua A. et al., 2012 : 16-17).

Obadias Garcia est le personnage central de la filière. Au long du Projet Waraná, il a su agréger autour de lui une diversité de soutiens interinstitutionnels et internationaux, depuis les anciennes autorités traditionnelles Sateré Mawé jusqu'aux entrepreneurs amazoniens ou européens (voir Figueroa A. L. 2016). Au départ du projet, Obadias convainc un producteur du fleuve Andirá, dont le site de production était situé sur le lieu actuel de l'écotourisme, de se joindre à lui et d'exporter $20 \mathrm{~kg}$ de guaraná en graines vers l'Italie. Le prix du guaraná fut alors de 20 euros le $\mathrm{kg}$ (120 francs à l'époque). Il passa à 30 euros en 2000, 40 en 2005, puis 50 euros le kg en 2011, soit, pour l'année 2015, un prix 10 à 20 fois supérieur au prix moyen brésilien, qui se situe autour de $10 \mathrm{R} \$$ le kg. Ainsi en 1995, $20 \mathrm{~kg}$ de graines de guaraná furent exportées vers l'Italie par la coopérative de commerce équitable CTM-Altromercato. Les graines furent transformées en extrait à Manaus, par un processus hydroalcoolique afin d'en faire une matière soluble dans une boisson gazeuse, par l'entreprise familiale Agrorisa. Le soda est lui, réalisé en Italie : c'est le Guaranito.

Photographie 2 : Début du partenariat entre CTM-Altromercato et le CGTSM.

De gauche à droite : Tuxaua Zuzu, Luiz Eusebi (CTSM) et Obadias Batista Garcia (1994)

Source : Nusoken 


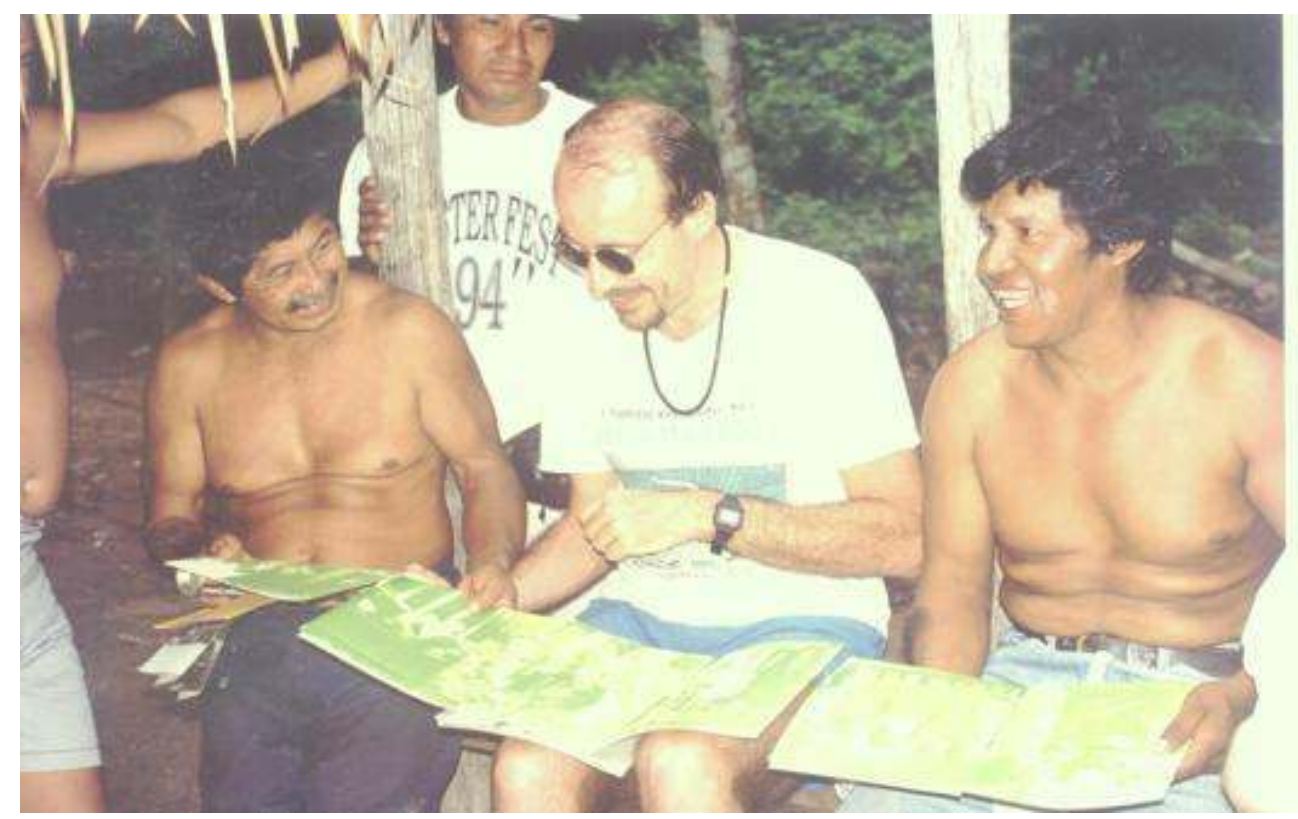

11 Un an plus tard, Claudie Ravel, une entrepreneuse ayant fondée une entreprise française de commerce équitable de produits écologiques originaires d'Amazonie et du Sri Lanka, la Société À Responsabilité Limitée (SARL) Guayapi, prend contact avec Obadias Garcia via Alba Figueroa, une anthropologue qui venait de terminer sa thèse de doctorat en anthropologie sur les Sateré Mawé à l'École des Hautes Études en Sciences Sociales (EHESS, voir Figueroa 2016 \& 1994). L'entreprise importa dès 1990 du guaraná biologique du Mato-Grosso et de Maués, mais voulait travailler avec le peuple autochtone qui avait découvert la plante et était prête à payer un surcoût pour appuyer un projet de développement selon les principes du commerce équitable et de l'agriculture biologique. Guayapi importa en poudre, du guaranà, une tonne en 1996. Le guaraná était valorisé sous forme de poudre $100 \%$ pure de graines de guaraná séchées sur les marchés de produits biologiques alors embryonnaires ${ }^{6}$. Dès le départ, le CGTSM en tant qu'association à visée politique, ne put pas exporter directement de produits vers l'étranger. Le CGTSM ne possédait ni moyens de transformation, ni fonds de roulement pour investir dans du capital de transformation. Cette tâche fut donc, jusqu'en 2008, confiée à une entreprise familiale de transformation et d'exportation de produits naturels d'Amazonie basée à Manaus, Agrorisa. Celle-ci joua le rôle d'intermédiaire entre les importateurs européens et le CGTSM, qui de son côté assura l'achat de guaraná au producteur et son acheminement jusqu'à l'usine. En 1998, les Sateré Mawé devinrent les pionniers de la certification biologique et en commerce équitable au Brésil avec l'organisme de certification Forest Garden Products (FGP) et la mise en place de techniques de Foresterie Analogue ${ }^{7}$ par le Docteur en écologie des systèmes sri-lankais Ranil Senanayake. En 2000, le Projet Guaraná fut présenté à l'Exposition Universelle d'Hanovre comme l'un des 146 projets exemplaires pour le futur, en tant que: "Satisfying Basic Needs Through the Exportation of a Tropical Fruit". En 2001, le «Waraná natif des Indiens Sateré Mawé » et le « Nectar des Abeilles Mélipones» sont reconnus comme produits d'excellence, et les Sateré Mawé producteurs, comme communautés de la nourriture par l'association slow Food International (voir Petrini C., 2011). 

(2001)

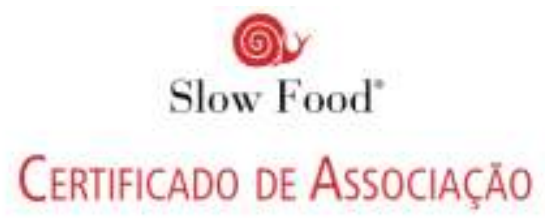

Certificamos que os produtores das Fortalezas do Waranà Nativo Sateré-Mawé e do Néctar de Abelhas Nativas săo membros do

\section{Convivium dos Filhos do Waraná e dos guardiōes do Jardim florestal do Imperador}

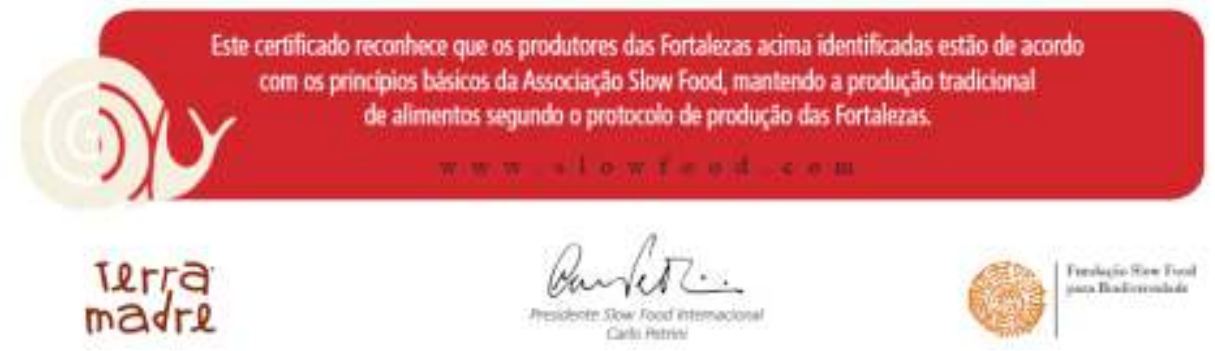

Le 19 décembre 2009, dans le village de Ilha Miquiles sur le fleuve Marau, est constitué le Consorcium des Producteurs Sateré Mawé (Consorcio dos Produtores Sateré Mawé, CPSM) dans le but de rendre autonome l'organisation productive de l'organisation politique des Sateré Mawé. Avant la création du CPSM le schéma d'exportation était le suivant (d'après Wolf S., 2012: 129-130). En octobre en Europe, les entreprises étrangères (Guayapi et CTM Altromercato) envoient en avance, comme cela se pratique dans le commerce équitable, la moitié de l'argent équivalente au volume de guarana qu'elles souhaitent acheter. Au même moment chez les Sateré Mawé, le guaraná fleuri se transforme en fruit et ne sera cueilli qu'à la fin du mois de novembre et début décembre. A ce moment, jusqu'en 2008, l'argent était réceptionné par une entreprise familiale à Manaus, Agrorisa, qui possédait l'autorisation d'exportation. En novembre, les leaders du CGTSM en charge du Projet Guaraná récupéraient l'argent auprès d'Agrorisa, pour qu'au mois de décembre ces derniers puissent voyager de communautés en communautés et acheter le guaraná aux producteurs à $40 \mathrm{R} \$$ le kilo. Ils ne payaient alors que la moitié du montant. En décembre-janvier, le guaraná en grain était envoyé à Manaus pour y être pulvérisé par Agrorisa. La poudre était ensuite mise en container et envoyée par bateau vers l'Europe. Une deuxième récolte à lieu en janvier et suit le même déroulé. En février, dès réception du guaraná en poudre en Europe, les entreprises importatrices envoient la deuxième moitié de l'argent à Agrorisa. En mars les leaders du CGTSM venaient à Manaus récupérer l'argent auprès d'Agrorisa et allaient distribuer de communauté en communauté l'argent restant aux producteurs. Le solde était gardé par le CGTSM pour les projets sociaux (récolte des déchets ${ }^{8}$, élevages d'abeilles natives, organisation d'assemblées...). Depuis 2008, le schéma s'est simplifié avec la creation du CPSM qui a vite obtenu des moyens de transformation et l'autorisation d'exporter sans passer par une organisation tierce. Les Sateré Mawé sont le premier peuple autochtone du Brésil à pouvoir exporter leur 
production de manière autonome avec le système Siscomex au Brésil. L'exportation suit donc le modèle suivant :

Graphique 1 : Chronomodèle actuel du Projet Waraná

Source : Blue Bees

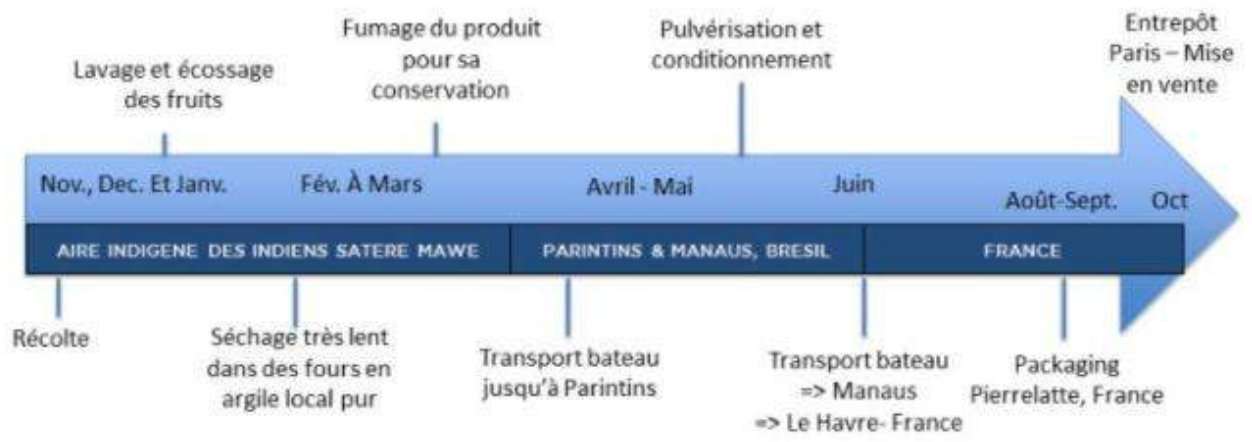

\section{Données économiques du Projet Waraná}

Dans la décennie des années 2000, nous pouvons avoir une meilleure idée des volumes de guaraná exportés par le CGTSM et le CPSM, de leurs valeurs et des montants perçus par les producteurs pour cela, comme le montre le tableau qui suit.

Tableau 1 : Volume et valeur des exportations de guaraná et montant alloué aux producteurs (2001-2013)

Source : CPSM

\begin{tabular}{|c|c|c|c|}
\hline Année de production & Volume de guaranà exporté (KG) & Chiffre d'affaires du CGTSM - CPSM & Part donnée aux producteurs (RS) \\
\hline 2001 & 9064 & 409043 & 165500 \\
\hline 2002 & 9045 & 769916 & 173200 \\
\hline 2003 & 6500 & 542837 & 130000 \\
\hline 2004 & 4589 & 618136 & 95720 \\
\hline 2005 & 7847 & 701659 & 156950 \\
\hline 2006 & 8960 & 739541 & 188200 \\
2007 & 5235 & 807684 & 157000 \\
2008 & 4927 & 822305 & 197000 \\
\hline 2009 & 5013 & 356039 & 200500 \\
\hline 2010 & 8642 & 1356794 & 345680 \\
\hline 2011 & 7512 & 1179384 & 507135 \\
\hline 2012 & 5857 & 919549 & 395406 \\
\hline 2013 & 2108 & 330956 & 142311 \\
\hline 2014 & 5722 & 898354 & 386292 \\
\hline
\end{tabular}

En 2010-2011, 434 familles de producteurs Sateré Mawé étaient enregistrées par le CPSM. Ainsi, le Projet Waraná amena pour cette récolte, une production moyenne en volume et en valeur de $20 \mathrm{~kg}$ de waraná pour 796,5 R\$ par an par producteur, soit environ 10 à $15 \%$ des revenus totaux des Sateré Mawé déclarés à partir des revenus de transfert des programmes sociaux de l'État brésilien. Cela est bien sûr à répartir avec les 5 enfants par femmes en moyenne enregistrés en 2004, ce qui indique, en supposant que chaque producteur fasse partie d'une famille nucléaire de 5 personnes, que le projet implique approximativement 2000 personnes, soit $1 / 6$ de la population totale Sateré Mawé. Le prix moyen payé par kg au producteur de guaraná se situe autour de $40 \mathrm{R} \$$ : soit 4 fois supérieur au prix moyen du guaraná payé au producteur au Brésil en 2010 estimé par l'IBGE à 8,36 R\$. Au sein de la Terre Indigène des Sateré Mawé, le fleuve 
Andirá compte de nombreux petits producteurs, de 10 à $90 \mathrm{~kg}$ de guaraná par an principalement, tandis que le fleuve Marau compte des plus gros producteurs de 100 à $600 \mathrm{~kg}$ par an principalement. La dichotomie entre la région du fleuve Marau, qui débouche à Maués, historiquement productrice et l'Andirá, où se trouve, à son embouchure vers le fleuve Boa Vista do Ramos, l'écotourisme de Vinte Quilos, est représentée dans la carte suivante. Cependant, comme l'analyse un ancien Sateré Mawé de l'Andirá,

Les Sateré ne pensent pas assez à leur futur (yan me). Les Sateré ne pensent pas assez à leur héritage et à leurs petits-enfants. Mais les tuxaua, eux, doivent penser au futur, afin d'honorer leur rôle d'autorités. Mais beaucoup n'y pensent pas non plus! Ils ne pensent pas : 'Diantre, aujourd'hui le guaraná a beaucoup de valeur!'. Personne ne pense à planter du guaraná. Et c'est à cause de cela que personne ne peut vendre 300, 400, $500 \mathrm{~kg}$. Aucun tuxaua n'annonce : 'Cette année, je vendrai tant de Quilos!'. Ils ne valorisent pas le prix du guaraná. Ils ne pensent pas à leur héritage. Je ne sais pas si les autres tuxaua ont planté du guaraná, mais moi je continue encore à planter du guaraná. Ici j'ai mon guaranazal, là, de l'autre côté, j'ai encore un autre guaranazal. De l'autre côté je vais encore l'agrandir. C'est ainsi que je parlais sur la question du guaraná. » (Tuxaua A. et al., 2012 : 17).

Carte 1 : Quantités de production de guaraná sur le fleuve Andirá (Terre Indigène Andirá-Marau, 2013)

Source : élaboration personnelle d'après le CPSM

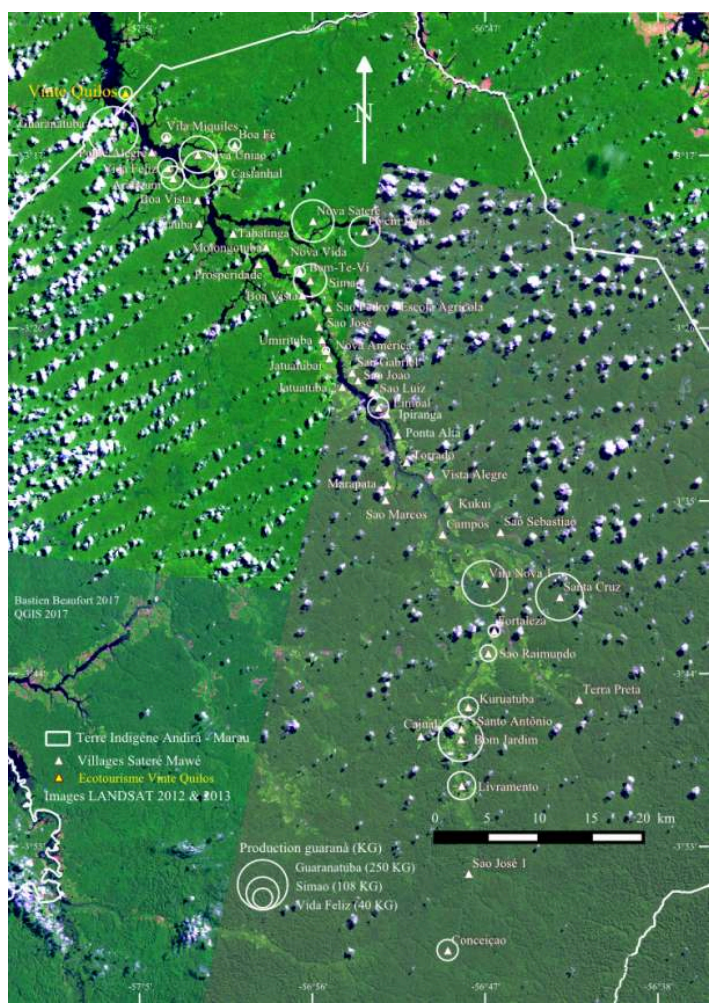

Carte : Quantités de production de guaraná sur le fleuve Marau (Terre Indigène Andirá-Marau, 2013)

Source : élaboration personnelle d'après le CPSM 


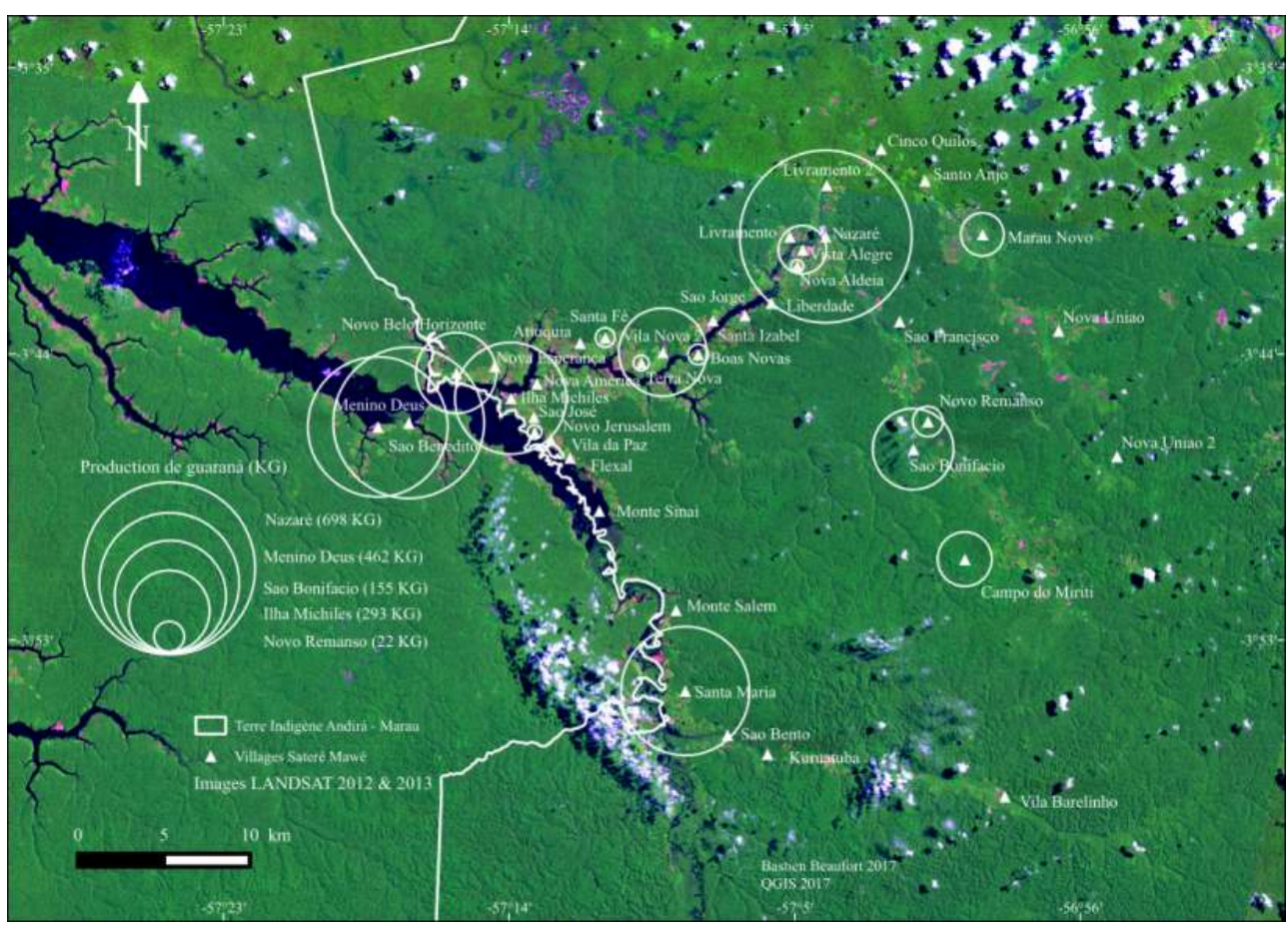

Le Consorcium des Producteurs Sateré Mawé (CPSM) se charge aujourd'hui d'acheter en avance le guaraná, ainsi que d'autres PFNL aux producteurs, afin de les transformer puis de les exporter ensuite vers l'Europe. Pour tous ces produits, la décomposition du prix, basée sur le guaraná, est réalisée de la manière suivante :

Tableau 2 : Décomposition du prix du kg de guaraná vendu par le CPSM en 2017

Source : Guayapi

\begin{tabular}{|c|c|c|}
\hline Nature & Montant $(€)$ & Pourcentage \\
\hline Prix d'achat aux producteurs & 20,73 & 41,46 \\
\hline Transformation / Conditionnement & 10,92 & 21,84 \\
\hline Capitalisation / Investissements & 1,7 & 3,4 \\
\hline Transport régional & 4 & 8 \\
\hline Accès au partage des avantages & 1,5 & 3 \\
\hline Appui au CGTSM pour l'ethnodéveloppement & 2,5 & 5 \\
\hline Promotion / Formation des cadres dirigeants / Consultations & 2,5 & 5 \\
\hline Certification / Impôts & 6,15 & 12,3 \\
\hline Cout de revient / Prix de vente total & 50 & 100 \\
\hline
\end{tabular}

15 En France, après la marge de Guayapi, puis de la boutique de revente au détail, le guaraná Sateré Mawé issu du Projet Guaraná se vend à un prix public de 25,5 euros les 65 grammes sous le nom « Waraná-Guaraná des Terres d'Origines », soit 392 euros le kg. Cela équivaut à environ $1000 \mathrm{R} \$$, soit une multiplication de la valeur créée au long de la filière, à partir du prix d'achat au producteur, de 25 à 33. À titre de comparaison les branches conventionnelles des filières du guaraná crééent une valeur équivalente à 300 fois le prix d'achat au producteur en moyenne (Beaufort B., 2017 : 390-392).

Tableau 3 : Décomposition du prix de vente généralement constaté auprès des consommateurs d'une boîte de Waraná - Guaraná des Terres d'Origine de marque Guayapi, Label "Sateré Mawé ", certifié Forest Garden Products (FGP) de $70 \mathrm{~g}$, en boutique spécialisée bioéquitable

Source : Guayapi 


\begin{tabular}{|c|c|c|}
\hline Nature & Montant (€) & Pourcentage \\
\hline Prix d'achat 70g & 3,4 & 14 \\
\hline Transport & 1,36 & 5,6 \\
\hline Manutention / Stockage & 1,7 & 7 \\
\hline Contenant & 0,36 & 1,5 \\
\hline Conditionnement & 0,34 & 1,4 \\
\hline Administration / Comptabilité & 1,7 & 7 \\
\hline Commercialisation & 3,88 & 16 \\
\hline Communication & 0,73 & 3 \\
\hline Charge détaillants & 9,46 & 39 \\
\hline TVA & 1,33 & 5,5 \\
\hline Prix public boite waranà & 23,25 & 100 \\
\hline
\end{tabular}

Une partie de l'argent du Projet Waraná est allouée à des projets d'ethnodéveloppement : c'est un des grands principes du commerce équitable tel que défini par l'Article 94 de la loi française sur l'Économie Sociale et Solidaire de 2014. En effet, le commerce équitable construit un prix d'achat fixe et supérieur aux producteurs, basé sur l'analyse des coûts de revient, incluant notamment une partie dédiée aux projets collectifs de développement. Les actes d'achats des citoyens sont donc considérés comme des actes politiques qui transforment graduellement et directement la société.

[...] la liberté de choix qu'offre la consommation constitue pour [les consommateurs] un champ d'affirmation d'une citoyenneté responsable, de sujets réflexifs et conscients de l'impact de leurs choix. Ces 'consom'acteurs' cherchent à la fois à défendre l'autonomie du consommateur en libérant l'acte de consommation de l'emprise des habitudes, du marketing et des lobbies, et souligner que cette liberté de choix du consommateur s'accompagne d'une responsabilité face aux conséquences sociales et environnementales de l'acte de consommer. Ils portent ainsi une vision alternative du sens et de l'exercice de cette liberté de consommer associant le renforcement de l'interdépendance au niveau de la planète et les défis environnementaux à la nécessité d'un auto-contrôle du consommateur. (Pleyers G., $2011: 17-18)$

En 2010-2011, 50 \% des producteurs vendirent moins de $9 \mathrm{~kg}$ de guaraná au CPSM et $75 \%$ moins de $19,5 \mathrm{~kg}$, soit des revenus inférieurs à $360 \mathrm{R} \$$ et $780 \mathrm{R} \$$ respectivement. Le commerce équitable du guaraná représente, pour les familles qui sont impliquées dans sa production, $1 / 5$ de leurs revenus totaux (voir Torres A. P. et al., 2010). Ce montant est supérieur aux analyses d'Ehringhaus à propos des revenus tirés de la commercialisation du caoutchouc et la noix d'Amazonie dans une Réserve extractiviste de l'Acre, au Brésil, qu'elle estimait représenter $5 \%$ des revenus totaux (Ehringhaus C., 2005 : 102). Cependant, cela est nettement moins que nos analyses sur l'économie du Projet agro-extractiviste (PAE) Chico-Mendes, en Acre aussi, où la noix d'Amazonie représente $80 \%$ des revenus moyens des familles estimés à un salaire minimum brésilien (Le Tourneau M. et Beaufort B., 2017). Cela confirme qu'un réel changement dans la vie des communautés forestières et paysannes ne pourra avoir lieu sans «se confronter aux élites nationales et aux conglomérats internationaux et en s'attaquant aux problèmes socio-économiques plus larges associés à la réforme foncière, l'économie et la politique commerciale. " (Ehringhaus C., 2005 : 107). Mais pour cela, les projets de commerce équitable, en renforçant les compétences organisationnelles des communautés de base, et en portant certains de leurs combats à une échelle nationale, voire globale, apparaissent comme un préalable efficace. Cette articulation entre l'échelle locale et l'échelle globale de l'action est l'idée historique des entreprises et associations engagées dans le commerce équitable et, partant, dans le tourisme équitable et 
solidaire : garantir que les producteurs-paysans (ou les hôtes, dans notre cas) puissent vivre dignement de leur travail, dans des environnements sains, et grâce à des produits et services exceptionnels, mais aussi transformer les règles du commerce et du tourisme mondial. Les principales conclusions des 77 études d'impacts commandées par le collectif Commerce Équitable France entre 2005 et 2015 vont dans ce sens : elles ont montré que les résultats positifs du commerce équitable n'étaient pas que monétaires ou économiques. Le commerce équitable a ainsi démontré avoir un impact sur la capacité des producteurs de s'auto-organiser pour définir et défendre leur autonomie ${ }^{9}$. Dans cet ordre d'idée d'encapacitation, en 2015, le Consorcium des Producteurs Sateré Mawé (CPSM) inaugura, dans la ville de Parintins, leur usine de transformation et d'exportation des produits issus de la Terre Indigène Andirá-Marau, dont le guaraná.

Photographie 4 : Inauguration de l'unité de transformation du CPSM à Parintins : Tuxaua (autorités traditionnelles) Adelino, Tiburcio et Eudes (2015)

Source : Nusoken

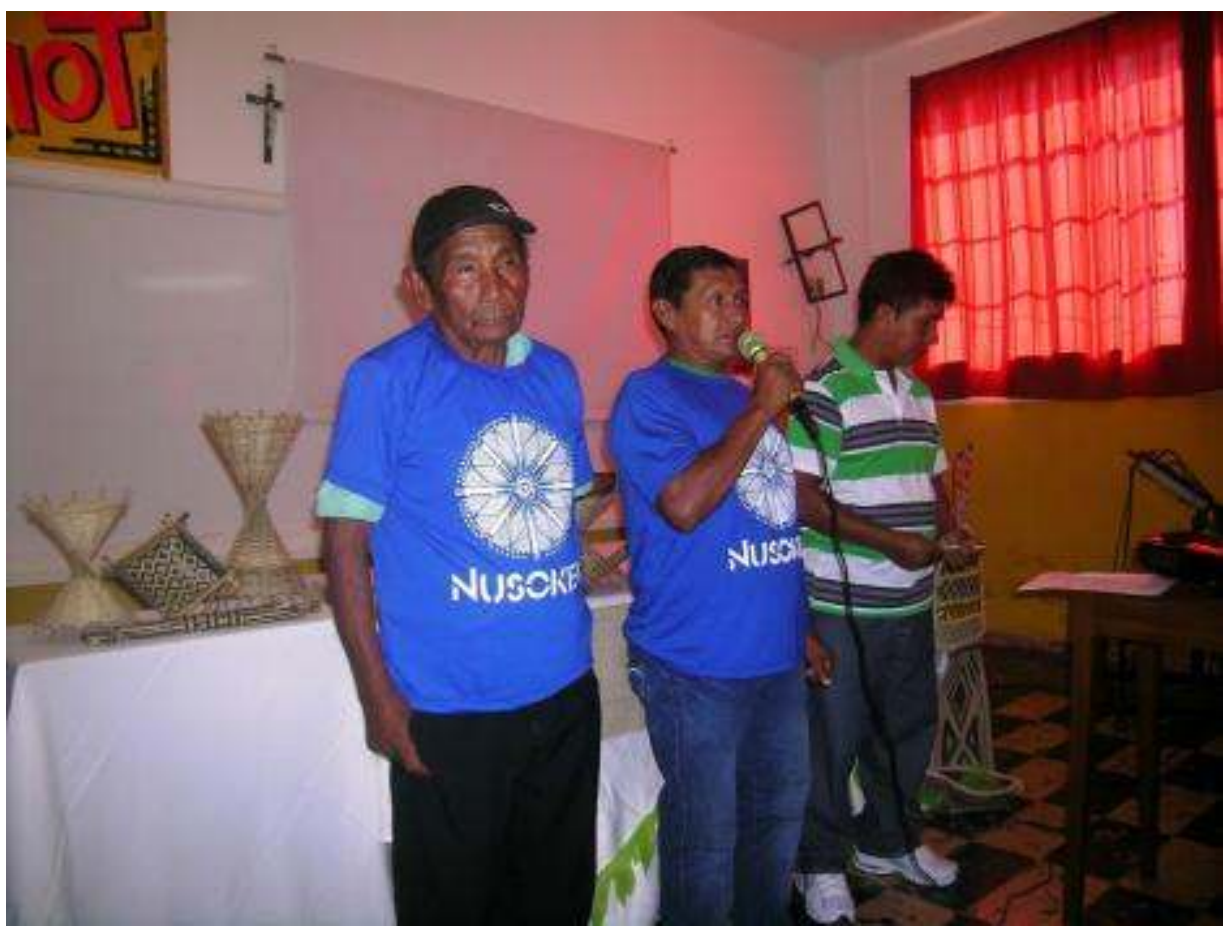

\section{L'écotourisme Vinte Quilos : une communauté modèle en attente d'activation?}

17 Le site d'écotourisme Vinte Quilos fait référence au lieu qui a vu se dérouler l'acte fondateur du Projet Waraná : l'exportation de $20 \mathrm{~kg}$ de guaraná Sateré Mawé sur les marchés du commerce équitable en Italie. Il est constitué comme une communauté modèle Sateré Mawé, englobant les aspects de la vie quotidienne : plantation de manioc et patates douces (roça), champs de guaraná polydiversifié (guaranazal consorciado), maison de séchage des graines du waraná dans les fours en argile (forno de barro), fumoir (fumeiro), mais aussi cuisine communautaire, énergie solaire, salle de conférence et bien sûr, maison d'accueil des touristes qui comprend 6 chambres au sein desquelles les 
(éco) touristes peuvent accrocher leurs hamacs. Dans le programme, des visites aux villages Sateré Mawé accessibles en bateau proche du site de Vinte Quilos sont prévues.

Photographies 5 à 9 : le site d'écotourisme Vinte Quilos (2010)

Source : personnelle
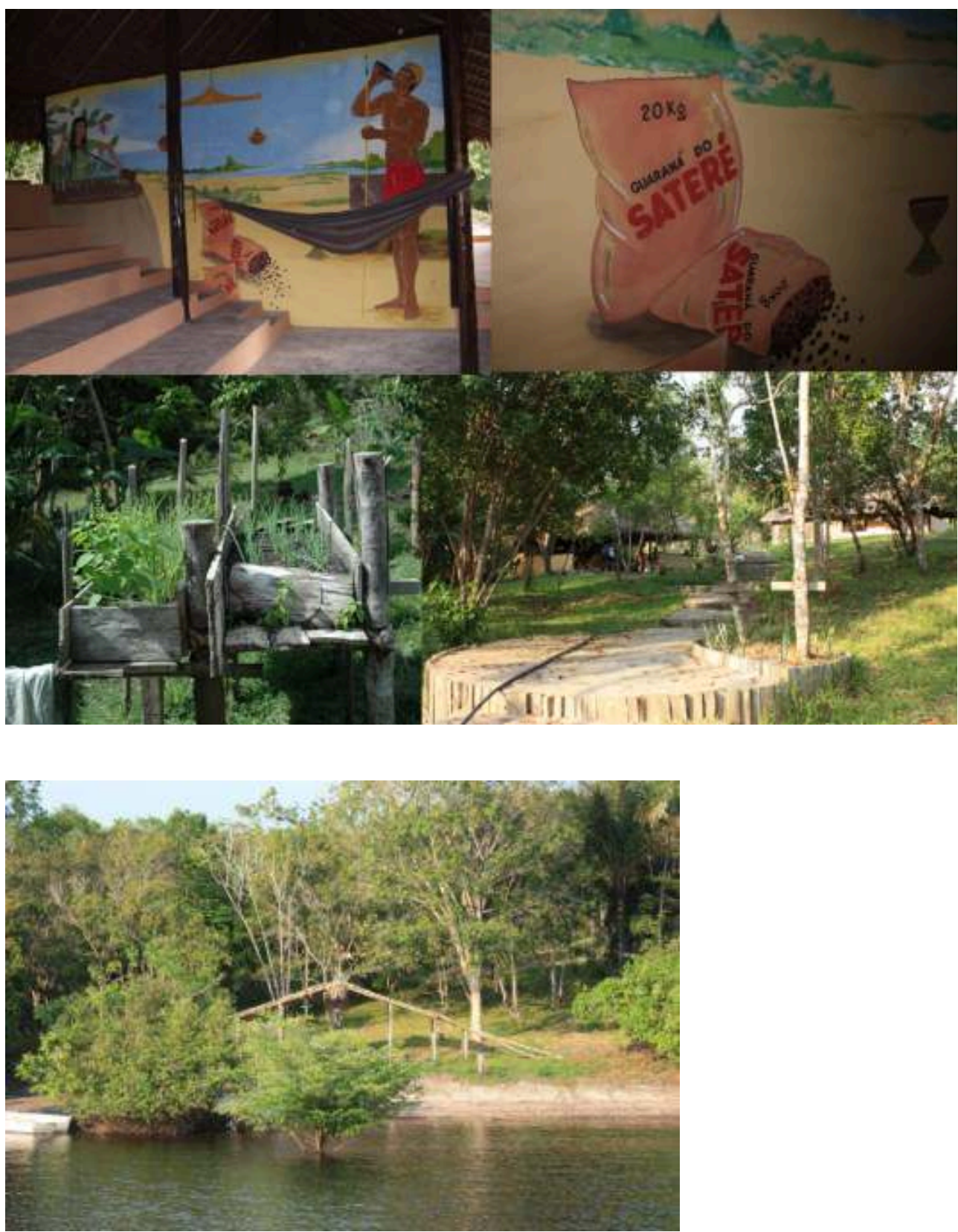

19 L'écotourisme Vinte Quilos reste cependant en attente d'activation, dans la mesure où il accueille ponctuellement des partenaires du Projet Waraná et non pas des écotouristes réguliers. Le Projet Waraná a besoin de grandir pour activer l'écotourisme de Vinte Quilos et lui donner une plus grande envergure, qui puisse être aussi une source de revenus pour les villages alentours. La localisation du site de Vinte Quilos, en bordure septentrionale de la Terre Indigène, symbolise le point de rencontre entre la 
société amérindienne et l'extérieur. Or, les Sateré Mawé sont en contact avec la société européenne depuis plus de 400 ans et cette friction est aussi intégrée mythologiquement dans le Projet Waraná avec le mythe de l'Empereur et le lieu du Nusoken.

\title{
Le mythe de l'Empereur: le futur des Sateré Mawé consiste à cultiver le jardin du Nusoken
}

La question historique du contact de plus de 400 ans avec la société environnante, de terrain d'entente commun et mouvant ou encore de friction interethnique des Sateré Mawé, s'exprime mythologiquement dans l'histoire de l'Empereur, qui représente «le fondement de l'intégration des blancs dans la cosmologie Sateré Mawé » (Wolf S., 2012 : 65). Le mythe relate, entre autres événements fondateurs, comment un Empereur a dit aux Sateré Mawé de s'installer sur les terres où ils sont aujourd'hui. Lors d'une réunion autour de la restitution d'une étude d'impact du commerce équitable chez les Sateré Mawé à Parintins en 2011 qui réunit des producteurs et Tuxaua Sateré Mawé ainsi que des organisations de commerce équitables européennes, un ancien leader Sateré Mawé, Lucio Menezes, prit la parole et interprèta le mythe de l'Empereur de la manière suivante :

\begin{abstract}
Les personnes de l'Empereur, ce sont nous. L'Empereur nous a dit où nous arrêter, pour que nous nous battions pour notre patrimoine, notre guaraná, avec tout le respect et l'honneur qui soient. Tout le monde connaît l'enfant d'où est né le premier Sateré, un enfant qui a été haï, maltraité, mais malgré cela, l'enfant a été enterré et ils dirent: 'Nous t'enterrons, tu naitras et seras la plus grande autorité du peuple Sateré Mawé. L'Empereur lui-même nous disait déjà, le fer volera dans le ciel. Un jour nos amis reviendraient et verraient nos besoins. Voilà Mauricio [le consultant du Projet Waraná de l'ONG Acopiama] qui est venu pour accompagner le projet. Combien de fois Mauricio a été critiqué ? Humilié ? Condamné ? Mais j'ai la certitude que l'Empereur dirait: 'Vous reviendrez par ici, pour emmener nos connaissances, notre guaraná, pour que notre guaraná ait la plus grande valeur. (Lucio Menezes, Parintins, cité dans Wolf S., 2012 : 65-66)
\end{abstract}

21 De la même manière, l'anthropologue Alba Figueroa, conclut sa thèse de doctorat dédiée aux Sateré Mawé sur ces paroles attribuées à l'Empereur dans le mythe raconté par Hernesto Cidade, qui exprime "[politiquement] un sentiment d'appartenance centré sur la terre, le lieu du milieu de leur cosmologie. La recherche de la Terre sans Mal n'est pas qu'un souvenir du passé.»: "Mes fils, vous pouvez rester car si nous partons tous, notre plantation demeurera sans personne pour s'en occuper. Si nous la laissons seule, qui s'en occupera? [...] Nous [les Sateré-Mawé] signifions, notre sens est d'être les gardiens de nos produits. » (Figueroa A. L., 1994 : 557). En référence directe au mythe de l'Empereur, le nom de domaine du site web du «Portail des Fils du Waraná », Nusoken (www.nusoken.com) reprend le nom du jardin de l'Empereur, qui est aussi le lieu du mythe de la naissance du guaraná : le jardin des pierres. C'est à partir de ce lieu, en son centre, que trouve son origine le matériau savoir du waraná et à partir de ce lieu, que se développeront toutes les ramifications du Projet Waraná. Dans cette dynamique, l'éducation différenciée est primordiale. Cette dernière passe aussi par la reconstitution des écosystèmes de la Terre Indigène Andirá-Marau, mise en place, dans le cadre du Projet Waraná, par la méthode de la Foresterie Analogue. 


\section{Le rôle de la Foresterie Analogue : restaurer les écosystèmes en imitant les forêts matures}

La Foresterie Analogue (Analog Forestry) est une méthode de sylviculture qui vise à imiter les fonctions écologiques et la structure architecturale des forêts matures. Initiée à la fin des années 1970 en Californie puis au Sri Lanka, la Foresterie Analogue a été mise en place en Terre Indigène Andirá-Marau avec les Indiens Sateré Mawé à partir de 1997 à l'initiative du Dr Ranil Senanayake, théoricien et practicien de la Foresterie Analogue. Cette dernière fut implémentée à travers la certification biologique Forest Garden Products (Produits de Jardins Forestiers, FGP) qui permet de garantir aux consommateurs que les produits qu'ils achètent, dans le cadre du Projet Waraná, viennent d'environnements sains et que les producteurs puissent être accompagnés dans leur organisation.

Photographie 10 : Foresterie Analogue à Vinte Quilos (2011)

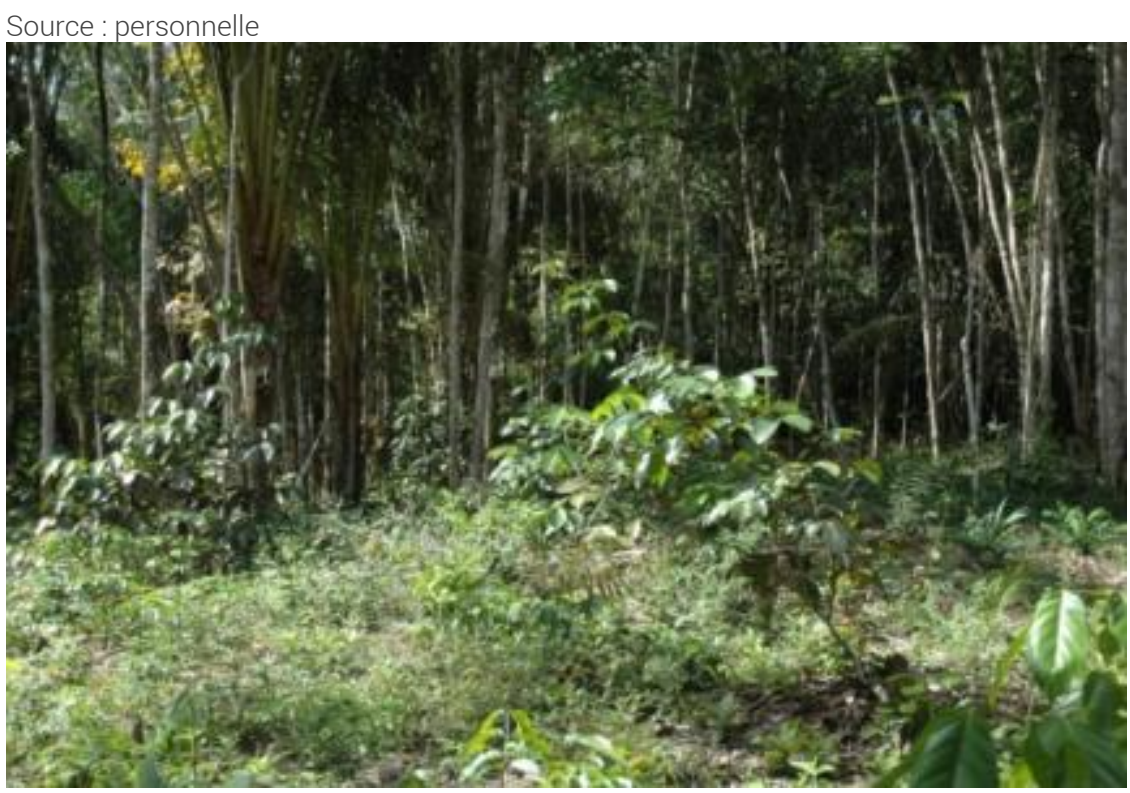

En conclusion, nous avons vu que l'écotourisme Vinte Quilo est une initiative unique en Amazonie dans la mesure où elle s'inscrit dans un projet international de commercialisation équitable de produits de la forêt par un peuple autochtone, les Indiens Sateré Mawé. Par ailleurs, ce projet international, le Projet Waraná, possède une inscription locale forte, car il vise à soutenir l'ethnodéveloppement intégré du peuple Sateré Mawé. La localisation de Vinte Quilos sur la bordure de la Terre Indigène, ouvrant vers l'Amazone et la ville de Parintins, est en ce sens symbolique ; tout comme son nom qui fait écho aux premiers $20 \mathrm{~kg}$ de guaraná exportés en 1993.

Cependant, Vinte Quilo reste un écotourisme qui n'est pas encore opérationnel, car peu de touristes responsables le visitent. Le site reste essentiellement visité par des membres des communautés Sateré Mawé et des partenaires directs du Projet Waraná, de manière ponctuelle. C'est pourquoi nous avons montré, au cours de cet article, comment Vinte Quilo est relié à l'histoire récente des Sateré Mawé, à l'organisation actuelle du Projet Waraná et aux actions menées pour le futur comme la Foresterie 
Analogue. Nous avons montré comment ces politiques s'inscrivent aussi dans la mythologie indigène et dans la relation particulière qu'entretiennent les Indiens Sateré Mawé avec la plante Waraná. De plus amples recherches futures, ainsi qu'un meilleur développement de Vinte Quilos, permettront de mieux comprendre les impacts de cet écotourisme amérindien en Amazonie brésilienne.

\section{BIBLIOGRAPHIE}

Beaufort, Bastien, La fabrique des plantes globales : une géographie de la mondialisation des végétaux du Nouveau Monde et particulièrement de l'Amazonie, Paris, Thèse de Doctorat de l'Université Paris 3 Sorbonne Nouvelle, 2017.

Boltanski, Luc \& Chiapello Eve, Le nouvel esprit du capitalisme, Paris, Gallimard, NRF « Essais », Paris, 1999.

Clement, Charles R. et al., "Origin and Domestication of Native Amazonian Plants", in Diversity, volume 2, 2010, pp. 72-106.

Congrétel, Mélanie, Une plante, des fils et des clones. Histoires amazoniennes de guaraná(s) dans un monde globalisé, Paris, Thèse de doctorat en sciences sociales, AgroParisTech, 2017.

Debuyst, Frédéric, «Limites et complémentarités d'une socio-économie associative » dans Auroi C. \& Yépez Del Castillo I., Économie Solidaire et Commerce Équitable. Acteurs et actrices d'Europe et d'Amérique latine, UCL Presses Universitaires de Louvain, Louvain, 2006, pp. 17-38.

Dreyfus-Gamelon, Simone, « Les Sateré Mawé et les Mundurucu : l'affaire Elf-Aquitaine », in Journal de la Société des Américanistes, tome 70, pp. 181-185, 1984.

Ehringhaus Christiane, Post-Victory Dilemnas: Land Use, Development, and Social Movement in Amazonian Extractive Reserves, A Dissertation Presented to the Graduate School of Yale University, In Candidacy of the Degree of Doctor of Philosophy, 2005.

Figueroa, Alba Lucy Giraldo, « Guaraná, a máquina do tempo dos Sateré-Mawé » in Boletem do Museu do Pará Emílio Goeldi Ciencias Humanas, volume 11, n 1, Belém, janvier-avril 2016, pp. 55-85.

Le Tourneau, François-Michel, Les Yanomami du Brésil. Géographie d'un territoire amérindien, Paris, Belin, 2010.

Le Tourneau François-Michel \& Beaufort Bastien, "Exploring the boundaries of individual and collective land use management: institutional arrangements in the PAE Chico Mendes (Acre, Brazil)", in International Journal of the Commons, volume 11, n 1, pp. 70-96, 2017.

Matos, Maria do Socorro Pacó, O olhar das Mulheres Sateré Mawé sobre o lixo, Manaus, EDUA, 2016. Pereira, Nunes, Os Índios Maués, Rio de Janeiro, Edição da Organização Simões, Coleção "Rex", 1954.

Petrini Carlo, Terra Madre. Renouer avec la chaîne vertueuse de l'alimentation, Paris, Traduit par Laurent Palet, Alternatives, « Manifestô », Paris, (2011) [2009]. 
Pleyers, Geoffroy, «Consommation critique : des promesses de la modernité aux enjeux de l'âge global » in Pleyers G. (dir.), La consommation critique. Mouvements pour une alimentation responsable et solidaire, Paris, Desclée de Brower, « Solidarité et Société », 2011.

Torres, Arturo Palma, Zerbini, Fabiola \& Gomes, Rosemary, Pratiques et impacts du commerce équitable du guaraná chez les Sateré-Mawé, Plate-Forme pour le Commerce Équitable, Paris, 2010

Tuxaua Adelino, Kapfhammer, Wolfgang, Oliveira, de Ranulfo, Histórias e Mítos Sateré Mawé, Bom Jardim, Nusoken, 2012.

Wolf, Sébastien, Modifications du système politique Sateré Mawé contemporain, Paris, Mémoire de M2R en Anthropologie, Mention Ethnologie, EHESS, 2012.

\section{Sites Internet - Ressources}

Association pour le Tourisme Équitable et Solidaire (ATES), 2018 : www.tourismesolidaire.org (dernier accès 28/10/2018)

Commerce Équitable France, 2018 : www.commercequitable.org (dernier accès 28/10/2018)

Nusoken, 2018 : www.nusoken.com (dernier accès 28/10/2018)

\section{NOTES}

1. Voir Beaufort B., 2017 ; Congrétel M., 2017.

2. On retrouve dans ces valeurs l'opposition entre altermodernité et l'hypermodernité, telle que le sociologue Jean Foyer l'a saisie avec le cas emblématique du maïs. Ce dernier a pu classer une opposition entre d'un côté une éthique défendue par des multinationales privées côtées en bourse de la semence, des produits phytosanitaires (Monsanto, Syngenta) et, si elles forment bien une autre branche, en sont parfois entièrement solidaires, celles des big pharma (Sanofi, Bayer, Mercks), et d'un autre côté une éthique paysanne de la semence (2010).

3. Entre août 1981 et janvier 1983, l'entreprise Elf-Aquitaine - aujourd'hui Total - s'allia avec l'entreprise brésilienne Braselfa pour réaliser des prospections pétrolières sur le territoire historique des Indiens Sateré Mawé. Cela entraîna des dommages importants qui furent dénoncés par des anthropologues, meneurs Sateré Mawé et une ONG indigéniste, le CTI. Les Indiens attaquèrent en justice les entreprises en mars 1983 et obtinrent en dédommagement environs $20000 €$ ainsi que l'obligation, de la part du gouvernement brésilien, de démarquer leurs terres (Dreyfus-Gamelon S., 1984).

4. En effet si au niveau des villages les tuxaua (twisa) sont investis par la descendance paternelle ou désignés de facto par ces derniers, les tuxaua élisent leurs représentants au sein du CGTSM.

5. Dont nous souhaitons ici honorer la mémoire et l'enseignement.

6. La firme distribue les produits Sateré Mawé sur près de 3000 boutiques spécialisées en agriculture biologique, commerce équitable et épicerie fine, en France et en Europe.

7. Méthode de sylviculture consistant à imiter la forêt primaire native dans ses fonctions écologiques et sa structure architecturale. Voir http://www.analogforestry.org/

8. Voir Maria do Socorro Pacó de Matos, O olhar das mulheres Sateré Mawé sobre o lixo (2016).

9. Études d'impact accessibles sur www.commercequitable.org dans l'onglet « Ressources ». 


\section{RÉSUMÉS}

Vinte Quilos est un site d'écotourisme mis en place au début des années 2000 en tant que vitrine du Projet Waraná, à l'initiative de meneurs Sateré Mawé, en Amazonie centrale brésilienne. Le Projet Waraná est un commerce alternatif de la plante waraná, plus connue internationalement comme le guaraná. Le Projet Waraná s'inscrit dans le mouvement mondial du commerce équitable et sa vitrine écotouristique, Vinte Quilos, dans celui du tourisme équitable et solidaire. $\mathrm{Au}$ cours de cet article nous présenterons le site d'écotourisme Vinte Quilos et nous expliquerons comment le Projet Waraná y est relié.

Vinte Quilos is an ecotouristic site established at the beginning of the 2000 as a showcase of the Waraná Project by Sateré Mawé indigenous leaders in the Central Amazon rainforest of Brazil. The Waraná Project is an alternative commerce of the waraná plant, internationally known as guaraná. The Waraná Project is part of the global fair trade movement and its ecotouristic showcase, Vinte Quilos, is part of the fair and solidarity tourism movement. During this article we will present the Vinte Quilos ecotouristic site and how the Waraná Project is related to it.

Vinte Quilos é um site ecoturístico estabelecido no inicio dos anos 2000 como vitrina do Projet Waraná por lideranças indígenas Sateré Mawé na Amazônia central do Brasil. o Projeto Waraná é um comercio alternativo da planta waraná, conhecida internacionalmente como guaraná. 0 Projeto Waraná faz parte do movimento global do comercio justo e sua vitrina ecoturística, Vinte Quilos, faz parte do movimento do turismo justo e solidário. Durante esse artigo estudaremos os differentes aspectos do Projeto Waraná e como o ecoturismo de Vinte Quilos esta relacionado ao projeto.

\section{INDEX}

Palavras-chave : waraná, ecoturismo, Amazônia, Sateré Mawé, Brasil

Mots-clés : Waraná, écotourisme, Amazonie, Sateré Mawé, Brésil

Keywords : Waraná, Brazil, ecotourism, Amazonia, Sateré Mawé

\section{AUTEUR}

\section{BASTIEN BEAUFORT}

Beaufort Bastien est docteur en géographie de l'IHEAL / CREDA, directeur adjoint de GUAYAPI, membre administrateur de l'IAFN-RIFA, président de Slow Food Paris Bastille et cofondateur de Disco Soupe. 55 rue Traversière 75012 Paris, bastienbeaufort@gmail.com 